\title{
Increased ex vivo cell death of central memory CD4 T cells in treated HIV infected individuals with unsatisfactory immune recovery
}

Marta Massanella ${ }^{1,4}$, Elisabet Gómez-Mora ${ }^{1}$, Jorge Carrillo ${ }^{1}$, Marta Curriu' ${ }^{1}$, Dan Ouchi ${ }^{1}$, Jordi Puig ${ }^{2}$, Eugènia Negredo ${ }^{2,3}$, Cecilia Cabrera', Bonaventura Clotet ${ }^{1,2,3}$ and Julià Blanco ${ }^{1,3^{*}}$

\begin{abstract}
Background: High levels of ex vivo CD4 T-cell death and the accumulation of highly differentiated and/or immunosenescent T cells have been associated with poor CD4 T-cell recovery in treated HIV-infected individuals. However, the relationship between cell death and T-cell differentiation is still unclear.

Methods: We have analyzed cell death, immunosenescence and differentiation parameters in HAART-treated subjects ( $V L<50$ copies/mL for more than 2 years) with CD4 T-cell count $<350$ cells/ $\mu \mathrm{L}$ (immunodiscordant, $n=23$ ) or $>400$ cells $/ \mu \mathrm{L}$ (immunoconcordant, $n=33$ ). We included 11 healthy individuals as reference.

Results: As expected, suboptimal CD4 T-cell recovery was associated with low frequencies of naive cells, high frequencies of transitional and effector memory cells and a subsequent low ratio of central/transitional memory cells in the CD4 compartment. These alterations correlated with spontaneous CD4 T-cell death. A deeper analysis of cell death in CD4 T-cell subsets showed increased cell death in memory cells of immunodiscordant individuals, mainly affecting central memory cells. Immunosenescence was also higher in immunodiscordant individuals albeit unrelated to cell death. The CD8 compartment was similar in both HIV-infected groups, except for an underrepresentation of naïve cells in immunodiscordant individuals.
\end{abstract}

Conclusion: Immunodiscordant individuals show alterations in memory CD4 T-cell differentiation associated with a short ex vivo lifespan of central memory cells and an in vivo low central/transitional memory cell ratio. These alterations may contribute to poor CD4 T-cell repopulation.

Keywords: Immunodiscordant, Cell death, T-cell subsets, Immunosenescence, HAART, cART

\section{Background}

HIV-1 infection leads to major perturbations of immune regulatory mechanisms [1] as a consequence of virusmediated depletion of CD4 T cells [2,3] and increased immune activation and inflammation [1]. Abnormal proliferative responses, maturation and immunosenescence of $\mathrm{T}$ cells, affecting both $\mathrm{CD} 4$ and $\mathrm{CD} 8$ compartments

\footnotetext{
*Correspondence: jblanco@irsicaixa.es

${ }^{1}$ Institut de Recerca de la Sida IrsiCaixa-HIVACAT, Institut d'Investigació en Ciències de la Salut Germans Trias i Pujol, Universitat Autònoma de Barcelona, 08916 Badalona, Spain

Full list of author information is available at the end of the article
}

and covering acute, chronic and late stages of infection have been reported [4].

Highly active antiretroviral therapy (HAART) efficiently controls HIV-1 replication, but inflammatory status and immune activation remain abnormally high in treated individuals compared with uninfected individuals [1]. Furthermore, CD4 T-cell recovery shows a heterogeneous response $[5,6]$; while some subjects reach CD4 T-cell counts comparable to healthy individuals, others fail to increase CD4 T-cell counts even after long-term ( $>5$ years) virologically effective treatment $[5,7]$. This immunodiscordant response has been associated with 
immunological damage induced by HIV-1 replication measured by nadir CD4 T-cell counts $[7,8]$ and specifically with the destruction of thymic tissue and the consequent lack of production of new naïve cells $[9,10]$. However, in the absence of new T cells, memory cells can repopulate the $\mathrm{CD} 4 \mathrm{~T}$-cell compartment in non-human primate models of HIV infection [11], suggesting that the damage of memory cells may also contribute to blunted immunological recoveries.

Increased replicative senescence, measured by the senescence marker CD57 [12], have been related with immune recovery [13] and could affect CD4 T cells in both naïve and memory subsets [14]. Apart from replicative senescence, skewed maturation of memory CD4 T cells [15] and higher turnover and sensitivity to spontaneous cell death $[9,15]$ may contribute to a lower proliferative potential of memory cells leading to incomplete immune recovery under HAART. However, it is still unclear how these different alterations of memory cells are linked and how are they associated with thymic damage or senescence. Moreover, the specific role of central memory CD4 $\mathrm{T}$ cells, a major player of HIV pathogenesis [16], in immune recovery remains poorly defined.

Considering the role of CD4 T-cell death in immunological recovery on HAART [6], we sought to analyze its relationship with altered maturation/immunosenescence profiles of memory cells. Therefore, we designed a study to compare these parameters in subjects with low and high CD4 T-cell recovery after long-term successful HAART. An aberrant maturation phenotype defined by a reduced central/transitional memory $\mathrm{CD} 4 \mathrm{~T}$-cell ratio, but not immunosenescence, strongly correlated with poor CD4 T-cell recovery and seems to be associated with high cell death levels in intermediate differentiation stages of memory cells.

\section{Methods Samples}

A cross-sectional study was performed to analyze the level of immunosenescence and differentiation of $\mathrm{T}$ cells in HAART-treated subjects. The Institutional Review Board of the Hospital Germans Trias i Pujol approved the study (EO code: EO-07-024) and all individuals provided written informed consent. Subjects that participated in the APOP- $\mathrm{V}^{+} \mathrm{I}^{-}$study $[8,17]$ were screened for the current study: 56 individuals with at least two frozen aliquots of PBMCs were selected. All subjects had confirmed diagnosis of HIV infection, continuous HAART with plasma viral load $<50$ copies $/ \mathrm{mL}$ for at least the last 2 years (minimum of 4 determinations during this period) and good treatment adherence. Exclusion criteria were: chemotherapy or interferon/ribavirin treatment and history of opportunistic infections during the last 2 years.

Patients were classified as Concordant (favorable virologic and immunologic response) when $\mathrm{CD} 4 \mathrm{~T}$-cell counts were above 400 cells $/ \mu \mathrm{L}$. Conversely, Discordant patients (favorable virologic but unsatisfactory immunologic response) showed CD4 $\mathrm{T}$ cells $<350$ cells $/ \mu \mathrm{L}$ $[8,17]$. Clinical and demographic data were collected from medical records. For comparative purposes, frozen PBMC from a control group of HIV uninfected individuals ( $\mathrm{n}=11$; EO-10-007, Institutional Review Board of the Hospital Germans Trias i Pujol) were also analyzed.

\section{CD4 and CD8 T-cell immunophenotype}

Frozen PBMCs were thawed at $37^{\circ} \mathrm{C}$, washed twice in $\mathrm{RPMI} / 20 \%$ of fetal bovine serum (FBS) and incubated for $1 \mathrm{~h}$ at $37^{\circ} \mathrm{C}$ before staining with a previously described eight-color panel [18] including CD3-APC-Cy7, CD4PerCP-Cy5.5, CD8-V500, CD57-FITC, CD27-APC, CD28-PE, CCR7-PE-Cy7 and CD45RA-V450 (BD Biosciences). Cell viability was evaluated by Propidium Iodide (PI, Sigma-Aldrich) staining in parallel tubes, the median [IQR] of $\mathrm{PI}^{+}$cells was 2.1\% [1.5-4.0]. Lymphocyte gate was defined by morphological parameters excluding most dead cells (less than $0.1 \%$ of $\mathrm{PI}^{+}$cells were found in the living lymphocyte gate). At least 300,000 lymphocytes were acquired in a LSRII cytometer (BD Biosciences) and analyzed with FlowJo software (Tree Star). Gating strategy is described in Additional file 1: Figure S1. Replicative senescence was defined by $\mathrm{CD} 57^{+} \mathrm{CD} 28^{-}$cells. T-cell subsets were first analyzed for CD27 and CD28 expression to identify Terminally Differentiated cells

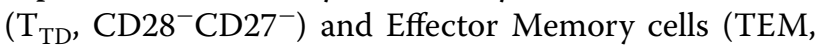
$\mathrm{CD} 27^{-} \mathrm{CD} 28^{+}$for $\mathrm{CD} 4 \mathrm{~T}$ cells or $\mathrm{CD} 27^{+} \mathrm{CD} 28^{-}$for $\mathrm{CD} 8$ $\mathrm{T}$ cells). Double positive $\mathrm{CD} 27^{+} \mathrm{CD} 28^{+}$cells were subanalyzed for CD45RA and CCR7 expression allowing for the definition of naive $\left(\mathrm{T}_{\mathrm{N}}, \mathrm{CD} 45 \mathrm{RA} \mathrm{CCR}^{+}\right)$, central memory $\left(\mathrm{T}_{\mathrm{CM}}, \mathrm{CD} 45 \mathrm{RA}^{-} \mathrm{CCR} 7^{+}\right)$and transitional

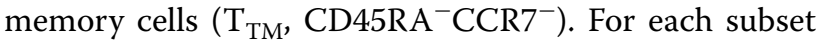
the expression of CD57 was analyzed (Additional file 1: Figure S1).

Immune activation, absolute lymphocyte counts (used to calculate absolute counts of each subset) and thymic production $\left(\mathrm{CD} 45 \mathrm{RA}^{+} \mathrm{CD} 31^{+} \mathrm{CD} 4 \mathrm{~T}\right.$ cells) were previously assessed in fresh blood samples in both CD4 and CD8 T cells $[8,17]$.

\section{Ex vivo culture of whole PBMC and sorted cells}

Data on spontaneous CD4 and CD8 T-cell death (including necrosis, intrinsic and extrinsic apoptosis) have been previously reported and were obtained after culture of freshly obtained PBMC for 24 h [17, 19]. Furthermore, 
in a subgroup of patients $(\mathrm{n}=10)$ freshly obtained PBMC were stained with CD3-APC-Cy7, CD4-PerCPCy5.5, CD8-V500, CD27-APC, CCR7-PE-Cy7 and CD45RA-V450 as described above, washed in PBS and sorted immediately in a FACSAria (BD Biosciences) to obtain gated $\mathrm{CD}^{+} \mathrm{CD}^{+}{ }^{+} \mathrm{CD} 8^{-}$cells with the following phenotypes: $\mathrm{T}_{\mathrm{N}}$ cells $\left(\mathrm{CD} 27^{+} \mathrm{CD} 45 \mathrm{RA}{ }^{+} \mathrm{CCR} 7^{+}\right)$, $\mathrm{T}_{\mathrm{CM}}$ cells $\left(\mathrm{CD}_{2} 7^{+} \mathrm{CD} 45 \mathrm{RA}^{-} \mathrm{CCR}^{+}\right), \mathrm{T}_{\mathrm{TM}}$ cells $\left(\mathrm{CD} 27^{+} \mathrm{CD} 45 \mathrm{RA}^{-} \mathrm{CCR} 7^{-}\right)$and a mixture of $\mathrm{T}_{\mathrm{EM}}$ and $\mathrm{T}_{\mathrm{TD}}$ cells $\left(\mathrm{T}_{\mathrm{EM}+\mathrm{TD}} \mathrm{CD} 7^{-}\right)$. These latter subsets were sorted as a single population due to technical limitations of sorting procedure (only 4 sorted populations could be obtained from a single sample). Sorted cells were cultured for $24 \mathrm{~h}$ and cell death was assayed using $40 \mathrm{nM}$ DIOC(6) as described previously [19]. DIOC(6) ${ }^{\text {low }}$ cells, having lost mitochondrial membrane potential, were identified as dead cells.

\section{Statistical analysis}

Continuous variables were expressed as the median (interquartile range) and compared using Mann-Whitney $\mathrm{U}$ test, permutation test for unbalanced groups or Signed-Rank test (for paired analyses). Discrete variables were described as percentages and compared using the Fisher's exact test. Correlations were assessed by the Spearman's test. Multiple comparisons were adjusted for false discovery rate. Statistical analyses were performed using $\mathrm{R}$ software version 3.0.2 [20] with two-tailed significance levels of $5 \%$.

\section{Results}

\section{Subject characteristics}

Subjects recruited in this study were stratified according to the level of recovery of CD4 T cells after suppressive HAART, as immunodiscordant or immunoconcordant (cutoff value $350 \mathrm{CD} 4 \mathrm{~T}$ cells $/ \mu \mathrm{L}$ ). The time course of CD4 T-cell recovery for each group is shown in Figure 1a. At the time of sample analysis, the median CD4 T-cell count was 220 and 798 cells/ $\mu \mathrm{L}$ for immunodiscordant and immunoconcordant HIV-infected individuals respectively $(p<0.0001)$, reflecting the blunted dynamics of CD4 T-cell recovery of the former group. Absolute CD8 T-cell counts showed no differences between HIV-infected groups. Median length of HIV infection (from diagnosis) was 11.8 and 10.1 years $(p=\mathrm{ns}$ ), while median time on treatment was 11.2 and 5.2 years $(p=\mathrm{ns})$ for immunoconcordant or immunodiscordant subjects, respectively. Overall, immunodiscordant individuals showed the previously reported higher CD4 T-cell death and activation with significantly lower nadir CD4 T-cell counts $[8,17]$. However, some immunoconcordant individuals showed also low nadir values. To evaluate the effect of nadir on immune recovery, immunoconcordant
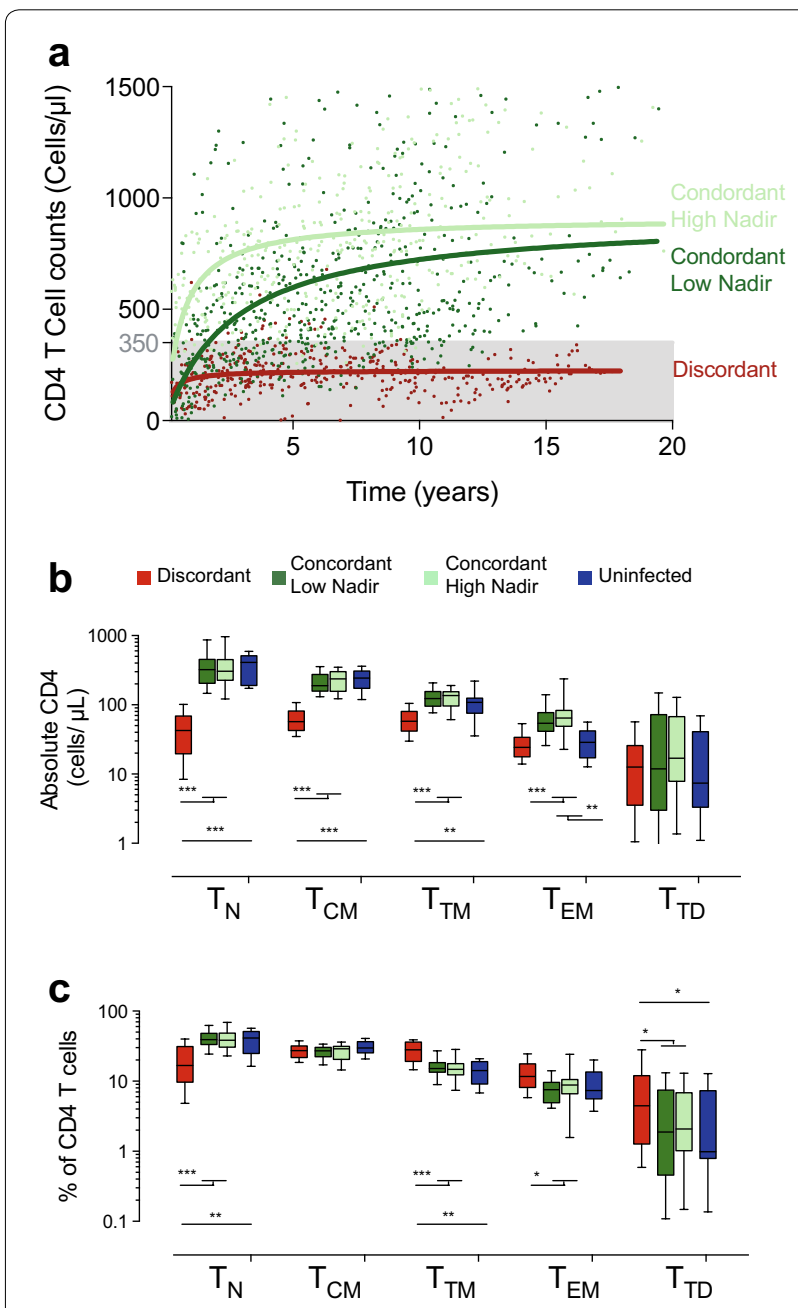

Figure 1 Characterization of CD4 T-cell subsets. a shows the evolution of CD4 T-cell counts in the three groups defined in this study (Immunodiscordant in red $(\mathrm{n}=23)$, immunoconcordant with low and high nadir in dark and light green, $m=17$ and $n=16$, respectively). Dots correspond to individual determinations of CD4 T-cell counts and lines show non-lineal regression of data plotted for comparative purposes. $\mathbf{b}$ The absolute count of circulating and $T_{N}, T_{C M}, T_{T M}, T_{E M}$ and $T_{T D}$ CD4 $T$ cells was analyzed in immunodiscordant individuals (red boxes), immunoconcordant individuals with low or high nadir (LN and HN; dark and light green boxes, respectively) and healthy HIV uninfected individuals ( $\mathrm{n}=11$, blue boxes). c The frequencies of the above-mentioned subsets in the CD4 T-cell compartment were also analyzed. In panels $\mathbf{b}$ and $\mathbf{c}$, data shown correspond to median values (band), IQR (boxes) and 10-90 interquartile values (whiskers). Figure show significant $p$ values (permutation test adjusted by false discovery rate): ${ }^{*} p<0.05 ;{ }^{* *} p<0.01$ and ${ }^{* * *} p<0.005$.

individuals were further divided according to the median nadir CD4 T-cell value (cut-off value $250 \mathrm{CD} 4 \mathrm{~T}$ cells/ $\mu L$ ) into two subgroups with low and high nadir values $(\mathrm{n}=17 \mathrm{ad} \mathrm{n}=16$, respectively). This allowed the direct comparison of immunoconcordant individuals with immunodiscordant subjects, avoiding the confounding 
effect of nadir and enabling us to evaluate the impact of low nadir values in immunoconcordant individuals. No major differences were observed among immunoconcordant subgroups, except for a higher presence of PIbased treatments and $\mathrm{HCV}$ co-infection in the low nadir group (Table 1).

\section{Analysis of the CD4 T-cell maturation}

Absolute counts and frequency of different CD4 T-cell subsets were analyzed in immunodiscordant, immunoconcordant (low and high-nadir subgroups) and 11 uninfected individuals. The data show that lower CD4 T-cell counts in immunodiscordant subjects (Figure 1a) were the consequence of lower levels of $\mathrm{T}_{\mathrm{N}}, \mathrm{T}_{\mathrm{CM}}, \mathrm{T}_{\mathrm{TM}}$ and
$\mathrm{T}_{\mathrm{EM}}$ cells compared with immunoconcordant individuals, while the absolute numbers of TTD cells were similar in all groups (Figure 1b). Interestingly, immunoconcordant patients, irrespective of the nadir values showed similar counts of all subsets that were in turn comparable to uninfected controls except for the $\mathrm{T}_{\mathrm{EM}}$ subset, suggesting a proper recovery of the CD4 T-cell subsets in these individuals (Figure 1b). The frequency of each subset in the CD4 T-cell compartment showed a significant underrepresentation of $\mathrm{TN}$ cells in immunodiscordant subjects (as compared to concordant or HIV-uninfected individuals) that was compensated by an overrepresentation of $\mathrm{T}_{\mathrm{TM}}$ cells and a less evident but still significant increase in $T_{E M}$ and $\mathrm{T}_{\mathrm{TD}}$ cells (Figure 1c). Conversely, $\mathrm{T}_{\mathrm{CM}}$ cells showed

Table 1 Main characteristics of the different groups

\begin{tabular}{|c|c|c|c|c|c|c|c|}
\hline & \multirow[t]{2}{*}{ Discordant $(n=23)$} & \multirow[t]{2}{*}{ a } & \multicolumn{3}{|l|}{ Concordant } & \multirow[t]{2}{*}{ b } & \multirow[t]{2}{*}{ Uninfected $(n=11)$} \\
\hline & & & All $(n=33)$ & Low Nadir $(n=17)$ & High Nadir $(n=16)$ & & \\
\hline Age (years), Median [IQR] & $48[45-50]$ & ns & $45[38-49]$ & $48[42-52]$ & $42[37-45]$ & ns & $38[34-47]$ \\
\hline Gender (\% of male) & 91 & ns & 85 & 76 & 94 & ns & 55 \\
\hline $\begin{array}{l}\text { Time since HIV diagnosis } \\
\text { (years), Median [IQR] }\end{array}$ & $10.1[4.1-20.4]$ & ns & $11.8[7.5-16.6]$ & $12.2[8.9-17.4]$ & $11.6[4.9-13.4]$ & ns & - \\
\hline $\begin{array}{l}\text { Time on HAART (years), } \\
\text { Median [IQR] }\end{array}$ & $5.2[3.5-11.4]$ & ns & $11.2[7.4-12.6]$ & $11.4[8.8-12.6]$ & $10.6[3.7-12.8]$ & ns & - \\
\hline Current HAART (\% PI-based) & 70 & $*$ & 33 & 41 & 25 & * & - \\
\hline HCV coinfection $(\%)$ & 35 & ns & 21 & 36 & 15 & * & 0 \\
\hline Ratio CD4/CD8, Median [IQR] & $0.23[0.17-0.33]$ & $*$ & $0.87[0.60-1.11]$ & $0.76[0.54-0.87]$ & $1.06[0.86-1.1]$ & ns & $1.64[1.31-1.81]$ \\
\hline $\begin{array}{l}\text { CD4 T cell counts (cells/ } / \mathrm{L} \text { ), } \\
\text { Median }[\mathrm{IQR}]\end{array}$ & 220 [192-253] & * & 798 [600-998] & 703 [600-896] & $881[672-1,075]$ & ns & $779[629-1,072]$ \\
\hline Nadir (cells/ $\mu \mathrm{L}$ ), Median [IQR] & $64[15-122]$ & * & $239[76-345]$ & 76 [19-185] & $351[280-429]$ & * & - \\
\hline $\begin{array}{l}\text { CD4 T-cell gain (cell/ } \mu \mathrm{L} / \text { year } \\
\text { HAART), Median [IQR] }\end{array}$ & $27[9-54]$ & * & $53[46-102]$ & $53[46-93]$ & $53[36-124]$ & ns & - \\
\hline $\begin{array}{l}\text { CD4 T cell (\% of lymph), } \\
\text { Median [IQR] }\end{array}$ & 14 [10-17] & * & 31 [27-39] & 29 [27-33] & $37[29-40]$ & ns & $42[36-45]$ \\
\hline $\begin{array}{l}\text { CD4 T-cell death (\%), Median } \\
\text { [IQR] }\end{array}$ & $9.3[7.5-15.1]$ & * & $4.6[3.3-5.9]$ & $4.9[4.4-5.6]$ & $4.3[2.9-6.1]$ & ns & $4.0[3.2-4.7]$ \\
\hline $\begin{array}{l}\mathrm{CD} 8^{+} \mathrm{CD}^{2} 5 \mathrm{RA}^{-} \text {(\% of CD4 T } \\
\text { cells), Median [IQR] }\end{array}$ & 37 [29-41] & * & 25.7 [20-32] & 26.5 [20-32] & $25.1[20-31]$ & ns & \\
\hline $\begin{array}{l}\mathrm{HLA}^{-D R^{+}} \mathrm{CD}^{+} 5^{+}(\% \text { of CD4 T } \\
\text { cells), Median [IQR] }\end{array}$ & $16[7.7-21.6]$ & * & $4.5[3.7-6.7]$ & $4.7[4.2-6.3]$ & $4.4[3.2-7.1]$ & ns & $2.0[1.5-2.7]$ \\
\hline $\begin{array}{l}\text { CD8 T cell counts (cells/ } \mu \mathrm{L} \text { ), } \\
\text { Median }[\mathrm{IQR}]\end{array}$ & $940[754-1,146]$ & ns & 908 [771-1,239] & $1,118[855-1,380]$ & $811[637-1,121]$ & ns & 459 [433-548] \\
\hline $\begin{array}{l}\text { CD8 T cell (\% of lymph), } \\
\text { Median [IQR] }\end{array}$ & $56[51-61]$ & * & 38 [34-46] & 39 [36-47] & $36[32-40]$ & ns & 24 [23-27] \\
\hline $\begin{array}{l}\text { CD8 T cell death (\%), Median } \\
\text { [IQR] }\end{array}$ & $7.1[4.8-10.3]$ & ns & $6.5[5.0-12.4]$ & $8.8[6.1-11.0]$ & $6.1[4.8-14.9]$ & ns & $3.9[3.4-4.9]$ \\
\hline $\begin{array}{l}\mathrm{CD}^{+} 8^{+} \mathrm{CD} 4 \mathrm{RA}^{-} \text {( } \% \text { of CD8 T } \\
\text { cells), Median [IQR] }\end{array}$ & $31[23-36]$ & * & 23 [19-35] & 25 [21-36] & 21 [19-26] & ns & $9[5-13]$ \\
\hline $\begin{array}{l}\mathrm{HLA}-\mathrm{DR}{ }^{+} \mathrm{CD}^{+} 5^{+}(\% \text { of CD8 T } \\
\text { cells), Median [IQR] }\end{array}$ & $12.6[6.9-22.4]$ & ns & $9.1[5.6-13.0]$ & $9.8[5.4-13.6]$ & $8.5[5.6-13.0]$ & ns & \\
\hline sCD14 ( $\mu \mathrm{g} / \mathrm{mL})$, Median [IQR] & $8.4[7.7-10.2]$ & ns & $8.8[7.2-9.7]$ & $9.2[7.6-10.1]$ & $8.0[7.1-9.2]$ & ns & $4.2[3.9-4.6]$ \\
\hline
\end{tabular}

$a$ Comparison of concordant and discordant subjects. ${ }^{*}$ denotes $p<0.05$; $n$ s non significant (Mann-Withney U or Fisher exact test).

$b$ Comparison of concordant subjects with low and high nadir. * denotes $\mathrm{p}<0.05$; ns non significant (Mann-Withney $\mathrm{U}$ or Fisher exact test). 
similar values in all groups. Again, both subgroups of immunoconcordant subjects showed similar values of subset frequencies reaching the levels of HIV-uninfected controls (Figure 1c).

\section{CD4 T-cell maturation and CD4 T-cell death}

In our previous studies we have shown that CD4 T-cell death, in particular intrinsic apoptosis, is a major determinant of immune recovery [8, 17]. Therefore, we explored the association of unbalanced CD4 T-cell maturation with the rate of cell death in ex vivo cultures of fresh PBMC. Spontaneous CD4 T-cell death was unrelated to the frequency of $C D 4 \mathrm{~T}_{\mathrm{CM}}$ or $\mathrm{T}_{\mathrm{TD}}$ cells but showed a significant negative correlation with the frequency of CD4 TN and positive correlation with $\mathrm{T}_{\mathrm{TM}}$ and $T_{E M}$ cells (Figure 2a). Since the frequency of CD4 $\mathrm{T}_{\mathrm{N}}$ and $\mathrm{T}_{\mathrm{TM}}$ cells were strongly inversely correlated (data not shown), we addressed independent associations by using a model including data from all subsets. This model (Additional file 2: Table S1) confirmed the independent positive association of $\mathrm{CD} 4 \mathrm{~T}$-cell death with the frequency of $\mathrm{T}_{\mathrm{TM}} \mathrm{CD} 4 \mathrm{~T}$ cells, clearly linking the higher presence of these cells with the increased cell death observed in immunodiscordant individuals.

However, these data do not discriminate whether TTM cell death rate is similar among treated HIV infected individuals or is higher in immunodiscordant patients. To experimentally confirm these possibilities, we addressed the analysis of the lifespan of $\mathrm{T}_{\mathrm{N}}, \mathrm{T}_{\mathrm{CM}}, \mathrm{T}_{\mathrm{TM}}$ and $\mathrm{T}_{\mathrm{EM}+\mathrm{TD}}$ CD4 T-cells from immunoconcordant and immunodiscordant individuals. Since PBMC culture did not allow for a direct assessment of cell death occurring in different CD4 T-cell subsets due to phenotypic changes upon cell death [21], we chose a previously reported sorting strategy prior to ex vivo culture to approach this issue [21]. Although this strategy may induce some additional stress to sorted cells, it allowed for a consistent measure of subset cell death (Figure 2b). In this set of experiments, we observed that TN cells showed significantly lower cell death rates than those observed in purified memory cells in both groups (Figure 2c). No significant difference in TN cell death was observed between groups. However, memory cells in immunodiscordant individuals showed higher rates of death (Figure 2c). This was associated with higher sensitivity to cell death in TCM cells from immunodiscordant individuals $(p=0.056)$, and non-significant trends in TTM and TEM ${ }_{+}$TD cells. Consistently, $\mathrm{T}_{\mathrm{N}}$ cell death did not correlate with CD4 T-cell counts while a significant negative association $(\mathrm{p}=0.013)$ was observed for $\mathrm{T}_{\mathrm{CM}}$ and trends were noticed for $\mathrm{T}_{\mathrm{TM}}$ and $\mathrm{T}_{\mathrm{EM}+\mathrm{TD}}$ cell death $(\mathrm{p}=0.067$ and 0.054 , respectively; Figure $2 \mathrm{~d})$. No significant correlations with senescence markers could be identified (data not shown).
To evaluate the impact of higher cell death rates on transition from $\mathrm{T}_{\mathrm{CM}}$ to more advanced stages of $\mathrm{CD} 4$ T-cell maturation, we analyzed the ratio between $T_{C M}$ and $\mathrm{T}_{\mathrm{TM}}$ cells. This parameter was significantly lower in immunodiscordant subjects compared to immunoconcordant (irrespective of nadir values) or uninfected individuals (Figure 3a). Interestingly, $\mathrm{T}_{\mathrm{CM}}$ to $\mathrm{T}_{\mathrm{TM}}$ transition is not completely normalized in immunoconcordant individuals (Figure 3a), suggesting that this parameter could be useful to assess the quality of immune recovery. Reinforcing the relevance of the $\mathrm{T}_{\mathrm{CM}} / \mathrm{T}_{\mathrm{TM}}$ ratio, a strong negative correlation was observed between this parameter and spontaneous CD4 T-cell death (Figure 3b). Overall, our data suggest that memory, but not $\mathrm{T}_{\mathrm{N}}$ cells, are major contributors to the increased cell death observed in immunodiscordant individuals [17]. Higher sensitivity to death of memory cell populations, especially $\mathrm{T}_{\mathrm{CM}}$, may help to explain the inability of immunodiscordant individuals to recover proper $\mathrm{T}_{\mathrm{CM}} / \mathrm{T}_{\mathrm{TM}}$ ratios.

\section{CD57 expression and immune recovery}

Besides $\mathrm{T}_{\mathrm{CM}}$ or $\mathrm{T}_{\mathrm{TM}}$ dysfunction, replicative immunosenescence of $\mathrm{CD} 4 \mathrm{~T}$ cells has also been related to immune recovery [14]. In our cohort, analysis of the frequency of $\mathrm{CD} 28^{-} \mathrm{CD} 57^{+} \mathrm{CD} 4 \mathrm{~T}$ cells (Additional file 1: Figure S1) showed higher levels in immunodiscordant subjects than in both immunoconcordant subgroups, which in turn showed similar levels to HIV-uninfected individuals (Figure 4a). The analysis of CD57 expression in the different CD4 T-cell subsets showed that despite apparent full immune recovery, immunoconcordant individuals displayed higher CD57 expression than uninfected controls in all CD4 T-cell subsets. In addition, immunodiscordant individuals showed highly significant differences with concordant subjects particularly in $\mathrm{T}_{\mathrm{N}}$ and $\mathrm{T}_{\mathrm{CM}}$ cells (Figure 4b).

We also evaluated the relationship of CD57 expression with CD4 T-cell survival. No significant correlation was found between replicative senescence $\left(\mathrm{CD} 28^{-} \mathrm{CD} 57^{+}\right.$cells) and spontaneous CD4 T-cell death (Figure 4c). These data suggest that different mechanisms regulate the expression of CD57 in different CD4 T-cell subsets and show that replicative senescence is not coupled with the spontaneous CD4 T-cell death rate characteristics of immunodiscordant responses to HAART.

\section{Analysis of the CD8 T-cell compartment}

Major comorbidities in HIV infected individuals have been related to CD8 T-cell activation $[1,22]$ and recently to the CD4/CD8 T-cell ratio. Therefore, we also evaluated differentiation and immunosenescence in CD8 T cells. Elevated CD8 T-cell counts in HIV infected individuals analyzed in this study (Table 1) were the consequence of 


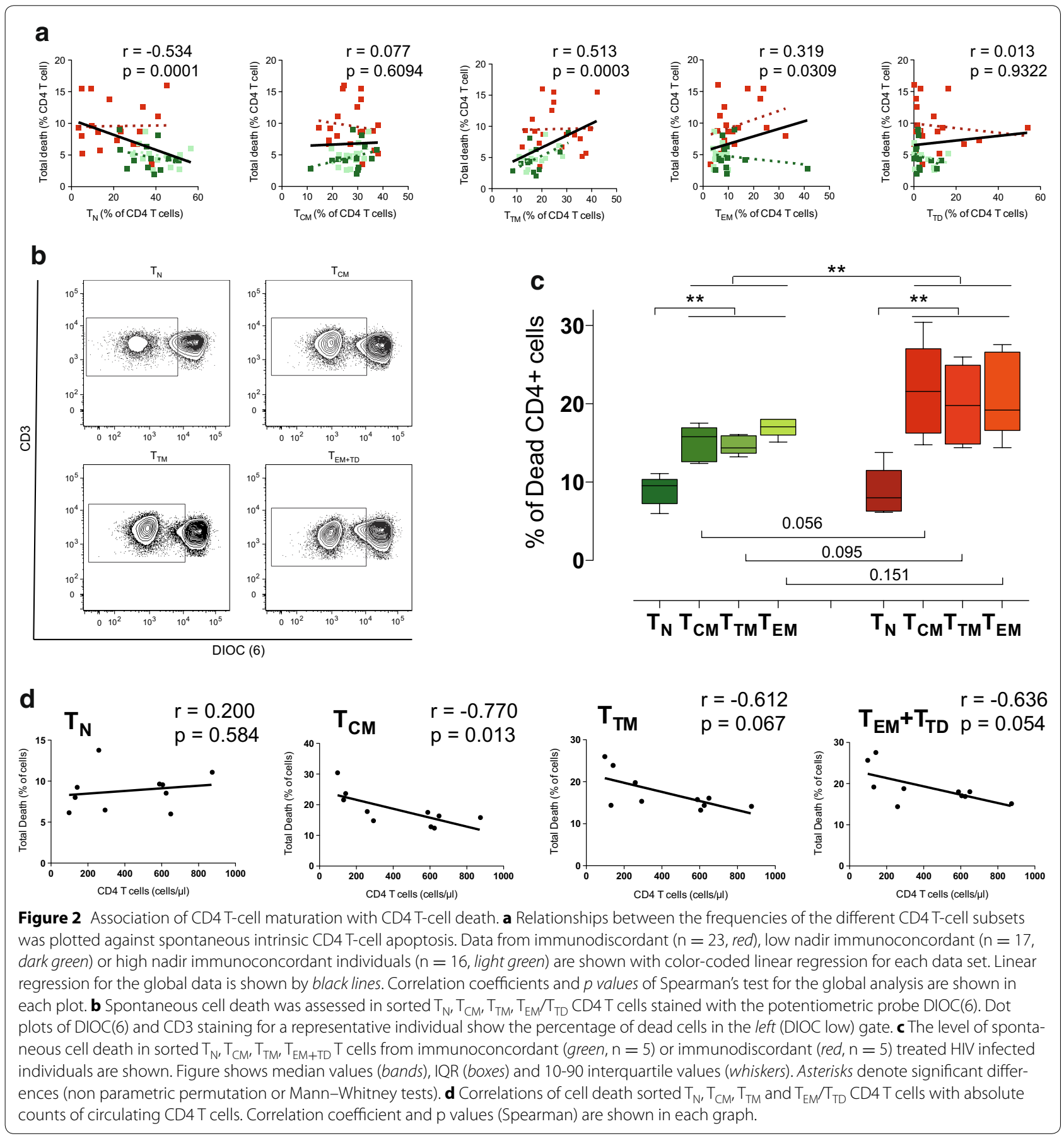

an expansion of all subsets except CD8 $\mathrm{T}_{\mathrm{CM}}$ cells (Figure 5a). Comparison of immunodiscordant and immunoconcordant subjects exclusively showed differences in the TN subset, consistent with the widely described thymic dysfunction of these patients [6]. The percentage of CD8 $\mathrm{T}$ cells was not normalized in immunoconcordant individuals, which showed intermediate values between immunodiscordant and uninfected subjects (Table 1).
Among immunoconcordant and immunodiscordant individuals, $\mathrm{T}_{\mathrm{TM}}, \mathrm{T}_{\mathrm{EM}}$ and $\mathrm{T}_{\mathrm{TD}} \mathrm{CD} 8 \mathrm{~T}$-cell subsets showed similar frequencies. However, a decreased size of the CD8 $\mathrm{T}_{\mathrm{N}}$ subset was observed in immunodiscordant individuals, while a reduced TCM subset was only observed in immunoconcordant subjects (Figure 5b). Replicative immunosenescence $\left(\mathrm{CD} 28^{-} \mathrm{CD} 57^{+}\right.$cells) was increased in both groups of HIV infected individuals 

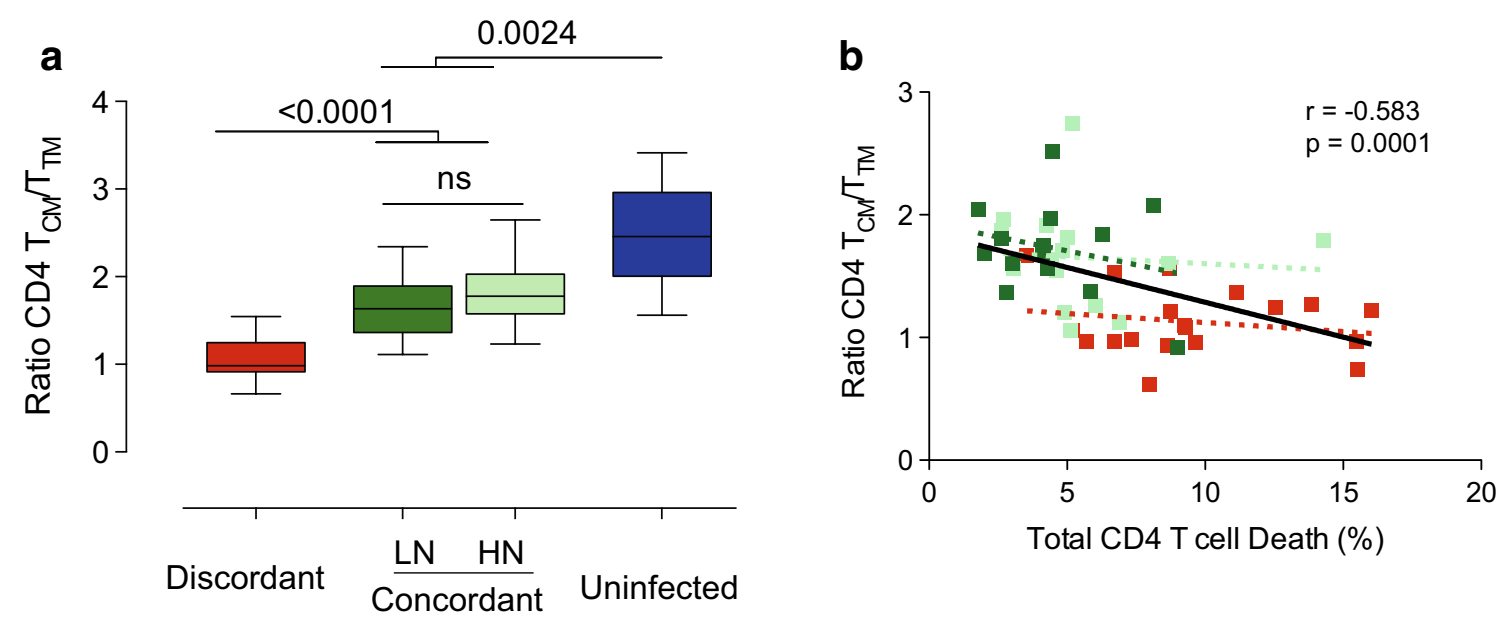

Figure 3 Ratio CD4 TCM/TTM and its association with total CD4 T-cell death. a The ratio between $T_{C M}$ and $T_{T M}$ cells was calculated as a measure of CD4 T-cell differentiation. Data shown correspond to median values (bands), IQR (boxes) and 10-90 interquartile values (whiskers) for immunodiscordant individuals ( $n=23$, red boxes), immunoconcordant individuals with low or high nadir ( $n=17$ and $n=16$, dark and light green boxes, respectively) and healthy HIV uninfected individuals $(n=11$, blue boxes). Figure shows significant $p$ values (permutation test adjusted by false discovery rate). b Correlation between total CD4 T-cell death and the ratio of CD4 $T_{C M} / T_{T M}$ cells. $P$ values for Spearman's test correlation are shown in black for all data points, and in red or green for immunodiscordant and immunoconcordant individuals respectively.

compared to uninfected controls; however, no differences between immunoconcordant and immunodiscordant individuals were observed. A deeper analysis of CD57 expression in the different CD8 T-cell subsets commonly showed a lower expression of CD57 in uninfected donors compared to HIV infected groups, with no differences between immunodiscordant and immunoconcordant individuals (Figure 5c). Again, we observed similar CD57 expression when this latter group was subdivided according to nadir values of CD4 T-cell counts. In summary, individuals displaying different CD4 T-cell recovery profiles seem to have similar CD8 T-cell compartments, except for the number of CD8 $\mathrm{T}_{\mathrm{N}}$ cells.

\section{Discussion}

Immunodiscordant responses to HAART are associated with high risk of mortality and the occurrence of AIDS and non AIDS-related diseases [23-28]. Although blunted CD4 T-cell recovery has been associated with impaired thymic output [29], other factors affecting memory cell populations, such as activation, replicative immunosenescence [30], skewed T-cell maturation [15, $30,31]$ and increased rates of CD4 T-cell death [8, 17], may contribute to persistently low CD4 T-cell counts.

The pivotal role of $\mathrm{TN}$ cells in CD4 T-cell repopulation and the lower size of this subset in immunodiscordant individuals has been universally reported $[5,10$, 32,33 ] and has been confirmed in our cohort using the CD31 marker $\left(C D 45 \mathrm{RA}^{+} \mathrm{CD} 31^{+}\right.$percentage of CD4 $\mathrm{T}$ cells) [17]. However, the thymus hypothesis should not overlook the role of memory cell populations, which may maintain T-cell homeostasis in thymectomyzed SIVinfected animals [11]. Immunodiscordant individuals show low proliferation of $T_{N}$ cells [15], suggesting that $T_{N}$ cells might be not required for $\mathrm{CD} 4 \mathrm{~T}$-cell repopulation (Additional file 3: Figure S2). In the absence of new CD4 TN cells, memory cells should be expanded by homeostatic mechanisms involving IL-7 or TCR stimulation to maintain CD4 T-cell numbers $[34,35]$. Since CD4 $\mathrm{T}_{\mathrm{CM}}$ cells are the main target of IL-7 [36] and IL-7 levels are increased in our cohort of immunodiscordant individuals [37], the inability to recover CD4 T cells, could also be related to the limited expansion capacity of memory cells. It could be argued that the CD4 TCM cell subset is under strong homeostatic pressure. Supporting this notion, high TCM proliferation have been reported in immunodiscordant individuals [15]. However, the limited size of this subset in immunodiscordant patients indicates that the proliferation of CD4 $\mathrm{T}_{\mathrm{CM}}$ cells may be inefficient or may result in increased maturation towards $\mathrm{T}_{\mathrm{TM}}$ or $\mathrm{T}_{\mathrm{EM}}$ phenotypes. This could explain the relative accumulation of CD4 T cells in intermediate stages of differentiation, mainly TTM but also TEM cells, observed in our cohort of immunodiscordant individuals. Interestingly, our data show an association between the levels of CD4 $\mathrm{T}_{\mathrm{TM}}$ cells and the rate of spontaneous CD4 T-cell death, although the analysis of cell death in sorted CD4 T-cell subsets in immunodiscordant and immunoconcordant individuals indicates that $\mathrm{CD} 4 \mathrm{~T}_{\mathrm{CM}}$ cell death is better correlated to CD4 T-cell recovery. Our data suggest that reduced $\mathrm{T}_{\mathrm{CM}}$ 


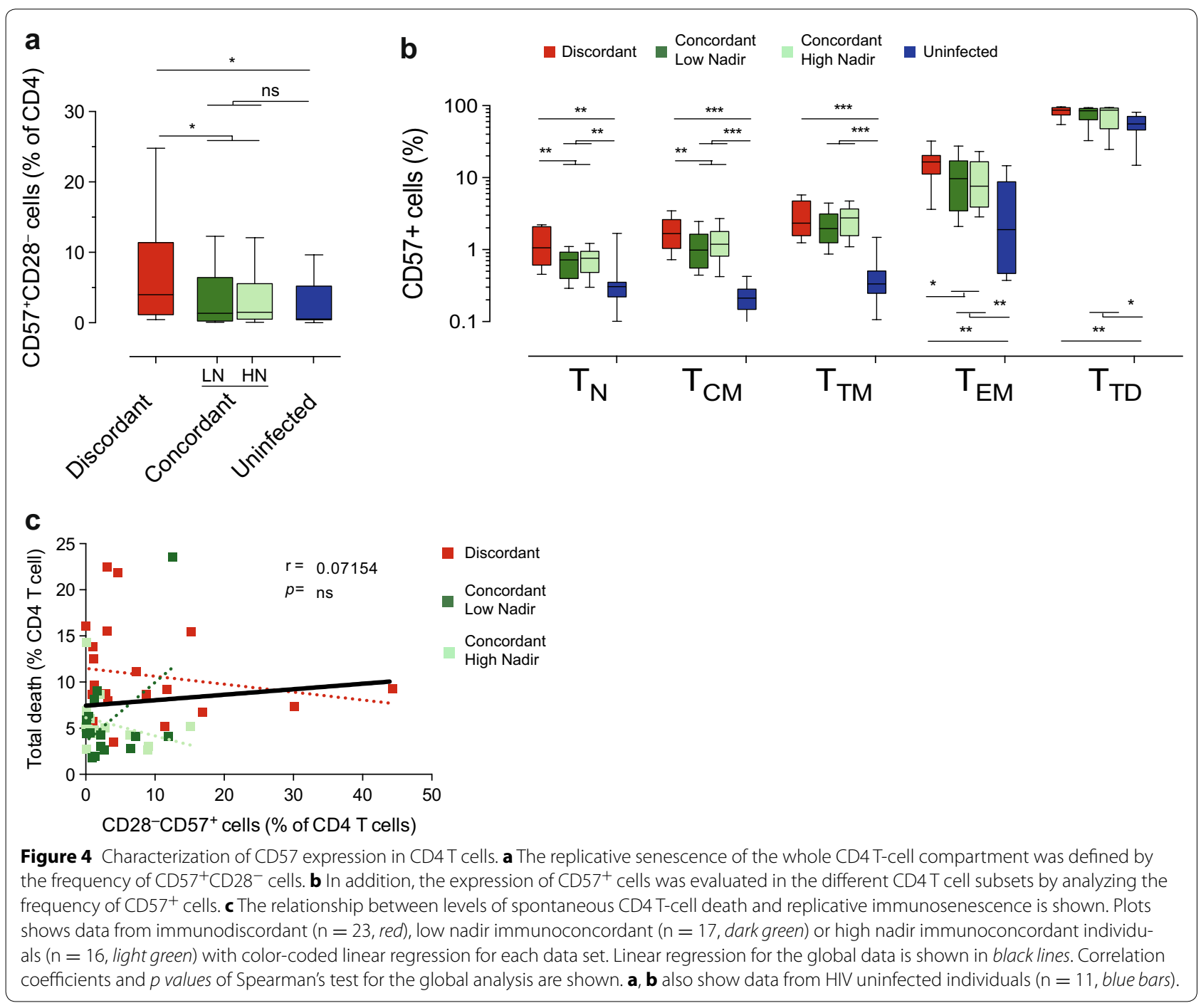

survival may be a second mechanism that adds to low thymic output in immunodiscordant patients.

An additional consequence of the high cell death level of TCM and the trends detected in $\mathrm{T}_{\mathrm{TM}}$ or $\mathrm{T}_{\mathrm{EM}}$ cells in immunodiscordant patients is the modest accumulation of senescent CD4 $\mathrm{T}$ cells. Since the fate of CD4 $\mathrm{T}_{\mathrm{CM}}$ and $\mathrm{T}_{\mathrm{TM}}$ or $\mathrm{T}_{\mathrm{EM}}$ could be cell death rather than further maturation (Additional file 3: Figure S2), this fact could explain the poor relationship between immunodiscordance and replicative senescence. Regarding senescence, our data on CD57 expression are consistent with previous reports [29], revealing higher levels of $\mathrm{CD} 28^{-} \mathrm{CD} 57^{+} \mathrm{CD} 4 \mathrm{~T}$ cells in immunodiscordant individuals. Although some reports suggest that $\mathrm{CD} 57^{+} \mathrm{CD} 4 \mathrm{~T}$-cells undergo faster spontaneous apoptosis [38], the frequency of these cells is not associated with cell death rate in the CD4 T-cell compartment, suggesting that they are unlikely to drive increased cell death in immunodiscordant individuals.
Consistently, several authors suggest that $\mathrm{CD} 28^{-} \mathrm{CD} 57^{+}$ cells are long lived $[39,40]$. Aside from replicative senescence, the expression of CD57 in other CD4 T-cell subsets, namely CD4 $\mathrm{T}_{\mathrm{N}}$ cells, has been also associated with CD4 T-cell recovery [30]. Although we have also noticed this association, it remains unclear whether $\mathrm{CD} 57^{+}$cells in $\mathrm{T}_{\mathrm{N}}$ and $\mathrm{T}_{\mathrm{CM}}$ subsets are actually senescent [12], or reflect other $\mathrm{T}$-cell functions or subsets such as Follicular helper CD4 T-cells ( $\left.\mathrm{T}_{\mathrm{Fh}}\right)$, which express CD57 [41] and are recovered after HAART [42]. The increased expression of CD57 in CD4 $\mathrm{T}_{\mathrm{N}}$ and $\mathrm{T}_{\mathrm{CM}}$ cells from immunodiscordant individuals points to alterations in these subsets and suggests again a role for $\mathrm{T}_{\mathrm{CM}}$ cells in CD4 T-cell recovery.

Our data point out that the level of destruction of the immune system, as measured by the nadir CD4 T-cell count, which has been widely associated with poor CD4 T-cell recovery [5], fail to fully identify 


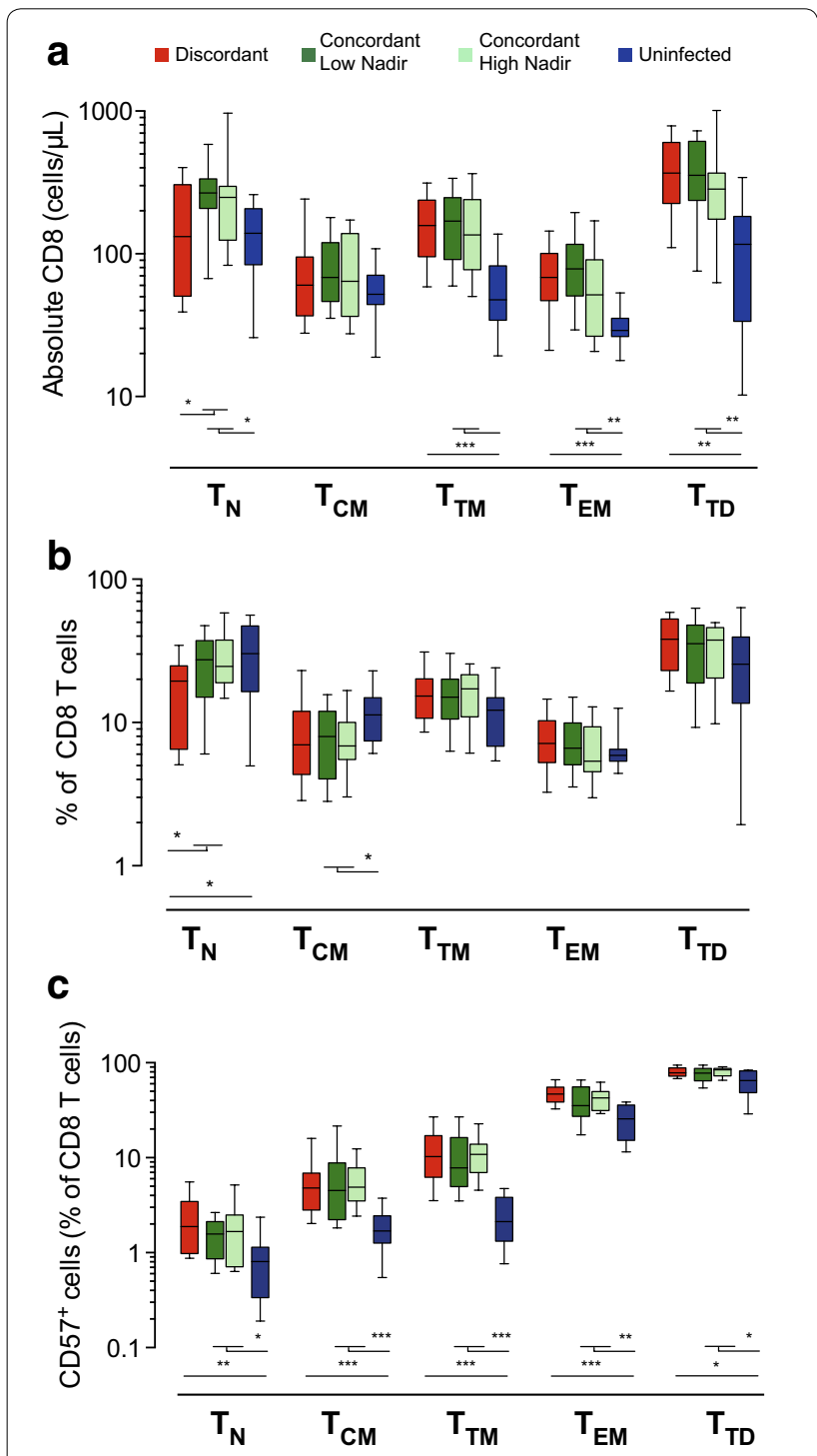

Figure $\mathbf{5}$ Characterization of CD8 T-cell subsets. The absolute number of circulating and $T_{N}, T_{C M}, T_{T M}, T_{E M}$ and $T_{T D}$ CD8 $T$ cells (a) was analyzed in immunodiscordant individuals ( $n=23$, red boxes), immunoconcordant individuals with low or high nadir ( $n=17$ and $n=16$, dark and light green boxes, respectively) and healthy HIV uninfected individuals ( $n=11$, blue boxes). The frequency of the above-mentioned subsets in the CD8 T cell compartment (b) was also analyzed. The expression of $\mathrm{CD}_{57}{ }^{+}$cells was further evaluated in the different subsets by the frequency of $\mathrm{CD}_{57}{ }^{+}$cells $(\mathbf{c})$. In all panels data shown correspond to median values (bands), IQR (boxes) and 10-90 interquartile values (whiskers) for immunodiscordant individuals (red boxes), immunoconcordant individuals with low or high nadir (green boxes) and healthy HIV uninfected individuals (blue boxes). Figure show $p$ values (permutation test adjusted by false discovery rate).

immunodiscordant responses. Indeed, some individuals with low nadir values are able to recover the CD4 T-cell compartment to the same extent as subjects with higher nadir values, at least in respect to maturation phenotypes. Although this observation does not occlude the fact that starting HAART with low CD4 T-cell counts increases the risk of immunodiscordant responses, it suggests that not only the number but also the quality of CD4 $\mathrm{T}$ cells determine immune reconstitution. In this regard, it is tempting to speculate that the size of the TCM compartment prior to HAART may yield a better predictive value [16].

We have also shown that despite the impairment/failure of thymic output in both CD4 and CD8 T-cell subsets, only CD4 $\mathrm{T}$ cells show an unbalanced maturation profile. While minimal differences were observed among HIV infected groups in CD8 T cells. This result is surprising since CD8 $\mathrm{T}$-cell senescence or activation has also been related to clinical progression in treated HIV infected individuals [43, 44]. Moreover, a recent analysis of HIV infected individuals failing to recover CD4/ CD8 T-cell ratios after HAART also points to maturation and activation of CD8 T cells as major markers of poor immune recovery [45]. This apparent paradox could be related to the different pressures acting on CD4 and CD8 $\mathrm{T}$ cells or to the specific features of our cohort, which exclusively included long-term treated individuals (more than 2 years) without AIDS defining events. It remains possible that we have selected individuals with low risk of clinical progression, potentially minimizing differences in CD8 T cells. On the other hand, our selection criteria and classification cutoff, based exclusively in CD4 T-cell counts, allows for the direct comparison of the CD4 T-cell compartment between groups, avoiding the confounding effects of inflammatory and clinical status. In contrast, our analysis has some limitations. First, inclusion criteria might select immunodiscordant patients with a trend towards a lower treatment time (see Table 1). However, correcting maturation and senescence analyses for time on treatment did not modify the main conclusions of the study (not shown). A second limitation comes from the definition of immunodiscordant responses. We have used a cutoff value of $350 \mathrm{CD} 4$ $\mathrm{T}$ cells/ $\mu \mathrm{L}$, while other authors define the limit in higher values. To assess the robustness of our analysis, a cutoff of $500 \mathrm{CD} 4 \mathrm{~T}$ cells/ $\mu \mathrm{L}$ has been also used, yielding similar results (data not shown). Finally, another confounding factor relevant in the context of this study is CMV co-infection, a major driver of immunosenescence. However, all HIV infected individuals and 9 out of 11 uninfected individuals recruited were seropositive for $\mathrm{CMV}$ infection. Exclusion of CMV seronegative controls did not modify our analysis.

\section{Conclusions}

In summary, our data point to a major perturbation of the central memory CD4 $\mathrm{T}$ cells as a new prominent 
characteristic of immunodiscordant responses to HAART. This perturbation adds to the increased accumulation of senescent cells and to the poor thymic output, and points to $\mathrm{CD} 4 \mathrm{~T}_{\mathrm{CM}}$ and $\mathrm{T}_{\mathrm{TM}}$ cells as the major players of CD4 $\mathrm{T}$-cell repopulation.

\section{Additional files}

Additional file 1: Figure S1. Gating strategy followed to identify T-cell maturation stages. Panel A. CD4 and CD8 T cells were gated and analyzed for the expression of CD27 and CD28. For CD4 T cells, terminally differentiated $\left(T_{T D}\right)$ cells were defined as CD28-CD27- ${ }^{-}$, while effector memory $\left(T_{E M}\right)$ cells were $C D 28^{+} C D 27^{-}$. Double positive cells were further analyzed for CCR7 and CD45RA expression to identify naïve $\left(T_{N}\right)$ cells $\left(C C R 7^{+} C D 45 R A^{+}\right)$, central memory $\left(T_{C M}\right)$ cells $\left(C C R 7^{+} C D 45 R A^{-}\right)$and transitional Memory $\left(\mathrm{T}_{\mathrm{TM}}\right)$ cells $\left(C C R 7^{-} \mathrm{CD} 4 \mathrm{RA}^{-}{ }^{-}\right.$). Panel B. For CD8 T cells, the general strategy was similar, unless for the definition of $\mathrm{T}_{\mathrm{EM}}$ cells that was $\mathrm{CD}_{27}{ }^{+} \mathrm{CD} 28^{-}$cells. The expression of $\mathrm{CD} 57$ was analyzed in the whole CD4 or CD8 T-cell population (lower left dot plot in each panel) to define replicative senescence (CD28- $\mathrm{CD} 57^{+}$cells). In addition the expression of CD57 in each subset was also evaluated (lower dot plots in each panels).

Additional file 2: Table S1. A. The multivariate relationship between $T_{N}, T_{C M}, T_{T M}, T_{E M}, T_{T D}$ and Total Death were determined by multiple linear regression. B. Linear regression model shows no statistical differences between group of patients (Discordant/Concordant) in the association of Total Death with $\mathrm{T}_{\mathrm{TM}}$.

Additional file 3: Figure S2. A model for CD4 homeostasis in Immunodiscordant individuals. Immunoconcordant individuals show a full recovery of CD4 T-cell counts with a high representation of naïve cells, and balanced frequencies of different memory subsets (upper panels). The profile of CD4 T-cell maturation in immunoconcordant individuals (green line) overlaps with that of HIV uninfected individuals (blue line in upper right plot) in which CCR7- ${ }^{-}$CD4 T cells show increased turnover and short live [22]. Conversely,immunodiscordant individuals (lower panels) show a reduced naiive subset with no signs of altered turnover [14] that limits the generation of new memory cells. Among memory subsets, central memory cells are also reduced and subjected to homeostatic pressure to generate new cells [14] increasing $\mathrm{T}_{\mathrm{CM}} \rightarrow \mathrm{T}_{\mathrm{TM}}$ transition (and lowering $\mathrm{T}_{\mathrm{CM}}$ ' $T_{T M}$ ratios) and increasing $T_{C M}$ cell death. $T_{T M}$ and $T_{E M}$ cells emerging from $T_{C M}$ cells also show higher sensitivity to cell death (Figure 2D), explaining the lack of accumulation of terminally differentiated cells in these subjects. This scenario results in a shifted profile of CD4 T-cell maturation (red line) compared to healthy individuals (blue line in lower right plot).

\section{Authors' contributions}

MM, MC, EG and JC performed flow cytometry data acquisition, analysis, and interpretation, and revised the manuscript; DO performed statistical data analysis; IP and CC contributed to data analysis and interpretation, and revised the manuscript; EN and BC contributed to study design, clinical follow up and revised the manuscript; IB contributed to study design and data interpretation and wrote the manuscript. All authors read and approved the final manuscript.

\section{Author details}

${ }^{1}$ Institut de Recerca de la Sida IrsiCaixa-HIVACAT, Institut d'Investigació en Ciències de la Salut Germans Trias i Pujol, Universitat Autònoma de Barcelona, 08916 Badalona, Spain. ${ }^{2}$ Fundació Lluita contra la SIDA, Institut d'Investigació en Ciències de la Salut Germans Trias i Pujol, 08916 Badalona, Spain. ${ }^{3}$ Universitat de Vic-Central de Catalunya, UVIC-UCC, 08500 Vic, Spain. ${ }^{4}$ Present Address: Department of Pathology, University of California San Diego, La Jolla, CA 92093, USA.

\section{Acknowledgements}

We are grateful to Leontina Banica (National Institute for Infectious Diseases, Bucharest) for critical reading of the manuscript, to technical staff of IrsiCaixa for sample processing and clinical staff of Fundació Lluita contra la Sida for clinical monitoring. This work was supported by the HIVACAT Program and the
Spanish AIDS network'Red Temática Cooperativa de Investigación en SIDA RD12/0017/0002 project as part of the Plan Nacional R + D + I and cofunded by ISCIII-Subdirección General de Evaluación y el Fondo Europeo de Desarrollo Regional (FEDER). J.B. and C. C. are researchers from Fundació Institut de Recerca en Ciències de la Salut Germans Trias i Pujol supported by the ISCIII and the Health Department of the Catalan Government (Generalitat de Catalunya). J.C. is supported by a 'Sara Borrell' grant from the Spanish Health Institute 'ISCIII'.

\section{Compliance with ethical guidelines}

\section{Competing interests}

All authors declare that they have no competing interests related to this work. Data have been partly presented at CROI meeting 2013.

Received: 9 April 2015 Accepted: 9 July 2015

Published online: 17 July 2015

\section{References}

1. Deeks SG (2011) HIV infection, inflammation, immunosenescence, and aging. Annu Rev Med 62:141-155

2. Blanco J, Barretina J, Cabrera C, Gutiérrez A, Clotet B, Esté JA (2001) $\mathrm{CD} 4(+)$ and $\mathrm{CD} 8(+) \mathrm{T}$ cell death during human immunodeficiency virus infection in vitro. Virology 285:356-365

3. Doitsh G, Cavrois M, Lassen KG, Zepeda O, Yang Z, Santiago ML et al (2010) Abortive HIV infection mediates CD4 T cell depletion and inflammation in human lymphoid tissue. Cell 143:789-801

4. Breton G, Chomont N, Takata H, Fromentin R, Ahlers J, Filali-Mouhim A et al (2013) Programmed death-1 is a marker for abnormal distribution of naive/memory T cell subsets in HIV-1 infection. J Immunol 191:2194-2204

5. Gazzola L, Tincati C, Bellistrì GM, Monforte A, Marchetti G (2009) The absence of $\mathrm{CD}^{+}{ }^{+} \mathrm{T}$ cell count recovery despite receipt of virologically suppressive highly active antiretroviral therapy: clinical risk, immunological gaps, and therapeutic options. Clin Infect Dis 48:328-337

6. Massanella M, Negredo E, Clotet B, Blanco J (2013) Immunodiscordant responses to HAART—mechanisms and consequences. Expert Rev Clin Immunol 9:1135-1149

7. Corbeau P, Reynes J (2011) Immune reconstitution under antiretroviral therapy: the new challenge in HIV-1 infection. Blood 117:5582-5590

8. Negredo E, Massanella M, Puig J, Pérez-Alvarez N, Gallego-Escuredo JM, Villarroya J et al (2010) Nadir CD4 T cell count as predictor and high CD4 $T$ cell intrinsic apoptosis as final mechanism of poor CD4 T cell recovery in virologically suppressed HIV-infected patients: clinical implications. Clin Infect Dis 50:1300-1308

9. Marchetti G, Gori A, Casabianca A, Magnani M, Franzetti F, Clerici M et al (2006) Comparative analysis of T-cell turnover and homeostatic parameters in HIV-infected patients with discordant immune-virological responses to HAART. AIDS 20:1727-1736

10. Ruiz-Mateos E, Rubio A, Vallejo A, De la Rosa R, Sanchez-Quijano A, Lissen E et al (2004) Thymic volume is associated independently with the magnitude of short- and long-term repopulation of CD4 ${ }^{+} T$ cells in HIV-infected adults after highly active antiretroviral therapy (HAART). Clin Exp Immunol 136:501-506

11. Okoye AA, Rohankhedkar M, Abana C, Pattenn A, Reyes M, Pexton C et al (2012) Naive T cells are dispensable for memory CD4 ${ }^{+}$T cell homeostasis in progressive simian immunodeficiency virus infection. J Exp Med 209:641-651

12. Brenchley JM, Karandikar NJ, Betts MR, Ambrozak DR, Hill BJ, Crotty LE et al (2003) Expression of CD57 defines replicative senescence and antigen-induced apoptotic death of CD8 ${ }^{+}$T cells. Blood 101:2711-2720

13. Fernandez S, Price P, McKinnon EJ, Nolan RC, French MA (2006) Low CD4 ${ }^{+}$ T-cell counts in HIV patients receiving effective antiretroviral therapy are associated with $\mathrm{CD}^{+}{ }^{+} \mathrm{T}$-cell activation and senescence but not with lower effector memory T-cell function. Clin Immunol 120:163-170

14. Méndez-Lagares G, Díaz L, Correa-Rocha R, León Leal JA, FerrandoMartínez S, Ruiz-Mateos E et al (2013) Specific patterns of CD4-associated 
immunosenescence in vertically HIV-infected subjects. Clin Microbiol Infect 19:558-565

15. Lederman MM, Calabrese L, Funderburg NT, Clagett B, Medvik K, Bonilla $\mathrm{H}$ et al (2011) Immunologic failure despite suppressive antiretroviral therapy is related to activation and turnover of memory CD4 cells. J Infect Dis 204:1217-1226

16. Okoye A, Meier-Schellersheim M, Brenchley JM, Hagen SI, Walker JM, Rohankhedkar M et al (2007) Progressive CD4 ${ }^{+}$central memory T cell decline results in $\mathrm{CD}^{+}{ }^{+}$effector memory insufficiency and overt disease in chronic SIV infection. J Exp Med 204:2171-2185

17. Massanella M, Negredo E, Pérez-Alvarez N, Puig J, Ruiz-Hernández R, Bofill $M$ et al (2010) CD4 T-cell hyperactivation and susceptibility to cell death determine poor CD4 T-cell recovery during suppressive HAART. AIDS 24:959-968

18. Curriu M, Carrillo J, Massanella M, Rigau J, Alegre J, Puig J et al (2013) Screening NK-, B- and T-cell phenotype and function in patients suffering from Chronic Fatigue Syndrome. J Transl Med 11:68

19. Massanella M, Curriu M, Carrillo J, Gómez E, Puig J, Navarro J et al (2013) Assessing main death pathways in T lymphocytes from HIV infected individuals. Cytometry A 83:648-658

20. R Core Team (2012) R: A language and environment for statistical computing. R Foundation for Statistical Computing, Vienna, Austria. ISBN 3-900051-07-0. http://www.R-project.org

21. Chirullo B, Sgarbanti R, Limongi D, Shytaj IL, Alvarez D, Das B et al (2013) A candidate anti-HIV reservoir compound, auranofin, exerts a selective 'antimemory' effect by exploiting the baseline oxidative status of lymphocytes. Cell Death Dis 4:e944

22. Deeks SG (2009) Immune dysfunction, inflammation, and accelerated aging in patients on antiretroviral therapy. Top HIV Med 17:118-123

23. Kaufmann GR, Furrer H, Ledergerber B, Perrin L, Opravil M, Vernazza P et al (2005) Swiss HIV Cohort Study: Characteristics, determinants, and clinical relevance of CD4 T cell recovery to $<500$ cells/microL in HIV type 1-infected individuals receiving potent antiretroviral therapy. Clin Infect Dis 41:361-372

24. Gutiérrez F, Padilla S, Masiá M, Iribarren JA, Moreno S, Viciana P et al (2008) Patients' characteristics and clinical implications of suboptimal CD4 T-cell gains after 1 year of successful antiretroviral therapy. Curr HIV Res 6:100-107

25. Piketty C, Weiss L, Thomas F, Mohamed AS, Belec L, Kazatchkine MD (2001) Long-term clinical outcome of human immunodeficiency virusinfected patients with discordant immunologic and virologic responses to a protease inhibitor-containing regimen. J Infect Dis 183:1328-1335

26. Young J, Psichogiou M, Meyer L, Ayayi S, Grabar S, Raffi F et al (2012) CD4 cell count and the risk of AIDS or death in HIV-Infected adults on combination antiretroviral therapy with a suppressed viral load: a longitudinal cohort study from COHERE. PLoS Med 9:e1001194

27. Tan R, Westfall AO, Willig JH, Mugavero MJ, Saag MS, Kaslow RA et al (2008) Clinical outcome of HIV-infected antiretroviral-naive patients with discordant immunologic and virologic responses to highly active antiretroviral therapy. J Acquir Immune Defic Syndr 47:553-558

28. Moore DM, Hogg RS, Chan K, Tyndall M, Yip B, Montaner JSG (2006) Disease progression in patients with virological suppression in response to HAART is associated with the degree of immunological response. AIDS 20:371-377

29. Appay V, Fastenackels S, Katlama C, Ait-Mohand H, Schneider L, Guihot A et al (2011) Old age and anti-cytomegalovirus immunity are associated with altered T-cell reconstitution in HIV-1-infected patients. AIDS 25:1813-1822

30. Méndez-Lagares G, García-Pergañeda A, del Mardel Pozo-Balado M, Genebat M, Ruiz-Mateos E, García García M et al (2012) Differential alterations of the CD4 and CD8 T cell subsets in HIV-infected patients on highly active antiretroviral therapy with low CD4 T cell restoration. J Antimicrob Chemother 67:1228-1237

31. Marchetti G, Gazzola L, Trabattoni D, Bai F, Ancona G, Ferraris L et al (2010) Skewed T-cell maturation and function in HIV-infected patients failing $\mathrm{CD}^{+}{ }^{+}$recovery upon long-term virologically suppressive HAART. AIDS 24:1455-1460

32. Solomon A, Cameron PU, Bailey M, Dunne AL, Crowe SM, Hoy JF et al (2003) Immunological and virological failure after antiretroviral therapy is associated with enhanced peripheral and thymic pathogenicity. J Infect Dis 187:1915-1923
33. Smith KY, Valdez H, Landay A, Spritzler J, Kessler HA, Connick E et al (2000) Thymic size and lymphocyte restoration in patients with human immunodeficiency virus infection after 48 weeks of zidovudine, lamivudine, and ritonavir therapy. J Infect Dis 181:141-147

34. Riou C, Yassine-Diab B, Van Grevenynghe J, Somogyi R, Greller LD, Gagnon D et al (2007) Convergence of TCR and cytokine signaling leads to FOXO3a phosphorylation and drives the survival of $\mathrm{CD}^{+}$central memory T cells. J Exp Med 204:79-91

35. Catalfamo M, Di Mascio M, Hu Z, Srinivasula S, Thaker V, Adelsberger J et al (2008) HIV infection-associated immune activation occurs by two distinct pathways that differentially affect CD4 and CD8 T cells. Proc Natl Acad Sci USA 105:19851-19856

36. Sereti I, Dunham RM, Spritzler J, Aga E, Proschan MA, Medvik K et al (2009) IL-7 administration drives T cell-cycle entry and expansion in HIV-1 infection. Blood 113:6304-6314

37. Massanella M, Ouchi D, Marfil S, Llibre JM, Puertas MC, Buzón MJ et al (2014) Different Plasma Markers of Inflammation Are Influenced by Immune Recovery and CART Composition or Intensification in Treated HIV Infected Individuals. PLoS One 9:e114142

38. Palmer BE, Blyveis N, Fontenot AP, Wilson CC (2005) Functional and phenotypic characterization of $\mathrm{CD} 57^{+} \mathrm{CD} 4^{+} \mathrm{T}$ cells and their association with HIV-1-induced T cell dysfunction. J Immunol 175:8415-8423

39. Spaulding C, Guo W, Effros RB (1999) Resistance to apoptosis in human $\mathrm{CD}^{+} \mathrm{T}$ cells that reach replicative senescence after multiple rounds of antigen-specific proliferation. Exp Gerontol 34:633-644

40. Gupta S (2005) Molecular mechanisms of apoptosis in the cells of the immune system in human aging. Immunol Rev 205:114-129

41. Bossaller L, Burger J, Draeger R, Grimbacher B, Knoth R, Plebani A et al (2006) ICOS deficiency is associated with a severe reduction of CXCR5 ${ }^{+}$CD4 germinal center Th cells. J Immunol 177:4927-4932

42. Boswell KL, Paris R, Boritz E, Ambrozak D, Yamamoto T, Darko S et al (2014) Loss of circulating CD4 T cells with B cell helper function during chronic HIV infection. PLoS Pathog 10:e1003853

43. Kaplan RC, Sinclair E, Landay AL, Lurain N, Sharrett AR, Gange SJ et al (2011) T cell activation predicts carotid artery stiffness among HIVinfected women. Atherosclerosis 217:207-213

44. Kaplan RC, Sinclair E, Landay AL, Lurain N, Sharrett AR, Gange SJ et al (2011) T cell activation and senescence predict subclinical carotid artery disease in HIV-infected women. J Infect Dis 203:452-463

45. Serrano-Villar S, Sainz T, Lee SA, Hunt PW, Sinclair E, Shacklett BL et al (2014) HIV-Infected Individuals with Low CD4/CD8 Ratio despite Effective Antiretroviral Therapy Exhibit Altered T Cell Subsets, Heightened CD8 ${ }^{+} \mathrm{T}$ Cell Activation, and Increased Risk of Non-AIDS Morbidity and Mortality. PLoS Pathog 10:e1004078

\section{Submit your next manuscript to BioMed Central and take full advantage of:}

- Convenient online submission

- Thorough peer review

- No space constraints or color figure charges

- Immediate publication on acceptance

- Inclusion in PubMed, CAS, Scopus and Google Scholar

- Research which is freely available for redistribution

Submit your manuscript at www.biomedcentral.com/submit
() Biomed Central 